\title{
Combination of lenalidomide and low-dose dexamethasone therapy promotes the anticoagulant activity of warfarin in patients with immunoglobulin light-chain amyloidosis
}

\author{
FUMIAKI KITAZAWA ${ }^{1}$, SHIN-ICHI FUCHIDA ${ }^{2}$, FUMITAKA ISE ${ }^{1}$, YOKO KADO ${ }^{1}$, \\ KUMI UEDA ${ }^{1}$, TAKATOSHI KOKUFU ${ }^{1}$, AKIRA OKANO ${ }^{2}$, MAYUMI HATSUSE ${ }^{2}$, \\ SATOSHI MURAKAMI ${ }^{2}$, YUKO NAKAYAMA ${ }^{3}$, KOHJI TAKARA $^{3}$ and CHIHIRO SHIMAZAKI ${ }^{2}$ \\ Departments of ${ }^{1}$ Pharmacy and ${ }^{2}$ Hematology, Japan Community Health Care Organization \\ Kyoto Kuramaguchi Medical Center, Kyoto, Kyoto 603-8151; ${ }^{3}$ Faculty of Pharmaceutical Sciences, \\ Himeji Dokkyo University, Himeji, Hyōgo 670-8524, Japan
}

Received August 19, 2015; Accepted February 23, 2017

DOI: 10.3892/ol.2017.6133

\begin{abstract}
The present study aimed to evaluate the drug interactions between warfarin and combination chemotherapy with lenalidomide and low-dose dexamethasone in immunoglobulin light-chain (AL) amyloidosis patients with unstable international normalized ratios (INR). The changes to INR values over time in $3 \mathrm{AL}$ amyloidosis patients treated with warfarin and a combination of lenalidomide and low-dose dexamethasone between March 2011 and February 2015 were analyzed retrospectively. The mean INR value was 1.52 prior to the combination chemotherapy, and the value increased 1.7-fold during treatment. The median time to reach maximum values was 17 days. Horn's drug Interaction Probability Scale indicated a possible interaction between lenalidomide and warfarin. These patients exhibited no marked alterations in hepatic function or serum albumin concentrations prior to and following combination chemotherapy and no additional administration of CYP2C9 inhibitors or vitamin K supplements was conducted. In addition, no patient experienced chemotherapy-induced nausea or appetite loss. These findings suggest that the total clearance or protein binding of warfarin remained unchanged. Therefore, the combination of warfarin and lenalidomide may cause a pharmacodynamic interaction, more likely by inhibiting the production of interleukin-6. In conclusion, clinically important interactions between warfarin and lenalidomide and low-dose dexamethasone therapy were observed in AL amyloidosis patients, where INR values signi ficantly increased. Therefore, close and regular monitoring of
\end{abstract}

Correspondence to: Professor Kohji Takara, Faculty of Pharmaceutical Sciences, Himeji Dokkyo University, 7-2-1 Kamiohno, Himeji, Hyōgo 670-8524, Japan

E-mail: takara@gm.himeji-du.ac.jp

Key words: lenalidomide, warfarin, drug interaction, pharmacodynamic, immunoglobulin light-chain amyloidosis patients during the course of treatment is important, and the dose of warfarin should be reduced if required.

\section{Introduction}

Immunoglobulin light-chain (AL) amyloidosis is a rare and potentially fatal disease characterized by clonal plasma cells in the bone marrow that produce abnormal $\kappa$ or $\lambda$ light chains (1). The mechanisms of outbreak, which arise from abnormal plasma cells in the bone marrow, are known to be similar to that of multiple myeloma (MM). The pathogenesis of MM is characterized primarily by the overproduction of interleukin-6 (IL-6) (2), the major growth and survival (anti-apoptotic) factor, which may also be expressed in AL amyloidosis (3).

Currently, there is no standard treatment for AL amyloidosis. However, lenalidomide (one of the more potent immunomodulatory drugs) and low-dose dexamethasone therapy has been approved for the treatment of AL amyloidosis (4) as this treatment has been demonstrated to be effective against MM (5), a condition with a similar pathology as AL amyloidosis. In addition, a common cause of mortality of patients with AL amyloidosis is cardiac dysfunction (6), including atrial fibrillation, a condition where warfarin is used to lower the risk of stroke.

Lenalidomide is excreted by the kidney, and the drug-metabolizing enzyme cytochrome P450 (CYP) is not involved in this metabolizing process $(7,8)$. Lenalidomide is known to be a poor substrate of P-glycoprotein (P-gp), but no clinically significant drug interactions between lenalidomide and P-gp substrates and inhibitors have been observed (9). Warfarin interacts with numerous drugs demonstrating pharmacokinetic or pharmacodynamic drug interactions (10-12). The pharmacokinetic interaction of warfarin is mediated by the inhibition of CYP2C9 or displacement of plasma protein binding. The pharmacodynamic interactions are primarily attributed to the additive or antagonistic effects on vitamin $\mathrm{K}$-dependent cycle of blood coagulation (13). Dexamethasone is an inducer of CYP3A4, an enzyme that metabolizes dexamethasone. The interaction between anticoagulant agents 
and corticosteroids including dexamethasone has been reported (14-16). It has been demonstrated that a high-dose of dexamethasone (40 mg/day for 4 days) is able to increase the international normalized ratio (INR) values in patients that take warfarin (14) and corticosteroids accelerated or inhibited anticoagulant activity $(15,16)$. In the case of low-dose dexamethasone, increase in INR elevation was not observed (17).

Recently, Weiss et al (18) demonstrated that co-administration of lenalidomide with a single dose of warfarin did not alter the plasma exposure or anticoagulant effect of warfarin or the plasma exposure of lenalidomide in healthy volunteers, but this was not the case for patients with overproduction of IL-6. However, it remains unclear whether there are interactions between multiple doses of warfarin and the combination of lenalidomide and low-dose dexamethasone.

The present study focused on evaluating the association of drug interactions between warfarin and the combination of lenalidomide and low-dose dexamethasone in patients with AL amyloidosis.

\section{Materials and methods}

Ethics approval. The present study was reviewed and approved by the Institutional Review Boards of the Japan Community Health care Organization Kyoto Kuramaguchi Medical Center (Kyoto, Japan; approval no. 2015012602). Written patient consent was waived since this was a retrospective and observational study.

Study population and design. A retrospective study was performed at the Japan Community Health care Organization Kyoto Kuramaguchi Medical Center (Kyoto, Japan). Initially, 4 patients with AL amyloidosis treated with 1.5-4.0 mg doses of warfarin and treated with a combination of lenalidomide and low-dose dexamethasone between March 2011 and February 2015 were included. However, 1 patient from this group was excluded from the assessment due to the increase of warfarin dose during lenalidomide and low-dose dexamethasone combination therapy.

The lenalidomide and low-dose dexamethasone regimen consisted of $15 \mathrm{mg}$ lenalidomide on days 1-21 and 12 or $40 \mathrm{mg}$ dexamethasone on days $1,8,15,22$ and 28 (28 days per cycle).

Data collection and evaluation. The anticoagulant activity of warfarin (INR values) was measured prior to and during lenalidomide and low-dose dexamethasone therapy. Throughout the combination chemotherapy, maximum INR value was obtained. Among the 6 cycles of chemotherapy for patients, the INR variation data for 4 cycles were analyzed. The other 2 cycles were excluded as the INR value prior to the combination chemotherapy was high as compared with that of the standard value (second cycle in Patient B), or warfarin was not administered prior to the combination chemotherapy (second cycle in Patient C). The dose of warfarin was stable throughout the combination chemotherapy.

Factors affecting the anticoagulant activity of warfarin, including additional administration of CYP2C9 inhibitors, hepatic function, serum albumin values, and chemotherapy-induced nausea and appetite loss were also assessed. Changes in these factors were compared prior and subsequent to the combination chemotherapy. Parameters of hepatic function, aspartate aminotransferase, glutamic-pyruvic transaminase and total bilirubin were reviewed.

The association of drug interaction between lenalidomide and warfarin were evaluated using Horn's Drug Interaction Probability Scale ( $>8$, highly probable; $5-8$, probable; $2-4$, possible; <2, doubtful) (19).

Statistical analysis. Data are expressed as the mean \pm standard deviation or median \pm range. A comparison of the INR values prior to the start of combination chemotherapy and during the course of the treatment was performed using the unpaired Student's t-test with Microsoft Excel software (version 2013; Microsoft Corporation, Redmond, WA, USA). P $<0.05$ was considered to indicate a statistically significant difference.

\section{Results}

Patient characteristics. The characteristics of patients enrolled in the present study are shown in Table I. All patients $(n=3)$ were diagnosed with AL amyloidosis and were administered warfarin due to cardiac dysfunction with atrial fibrillation. No patient exhibited significant alterations in hepatic function and serum albumin concentrations prior to and following combination chemotherapy. In addition, the patients were not treated with any other drugs possessing inhibitory activity of CYP2C9, except for warfarin. Furthermore, chemotherapy-induced nausea and appetite loss were not observed in any patient.

Clinical time course of INR values. Changes in INR values during the lenalidomide and dexamethasone combination therapy are shown in Fig. 1. In all cases, INR values were stable (mean, 1.52) prior to the chemotherapy. Although the dose of warfarin was unchanged, the INR value prior to combination chemotherapy was 1.69 in case 1 and increased to 2.66 on day 23 during chemotherapy. The INR value returned to 1.55 on day 50 during the withdrawal period.

Similar to the findings in case 1 , the INR values in case 2 increased from 1.56 to 2.80 on day 25 . In addition, the INR value markedly and quickly increased on day 4 of the second cycle from 2.96 to 4.03 due to re-administration of lenalidomide. On day 18 of the second cycle, INR values quickly increased to 2.23 , even though the dose of warfarin was reduced from 1.5 to $1.0 \mathrm{mg}$ /day and was only re-administered if lenalidomide was administered beforehand.

In case 3 , INR values increased from 1.56 to 2.64 on day 8 in the first cycle, resulting in discontinuation of warfarin. The dose of warfarin was reduced to half and was subsequently re-administered on day 2 of the second cycle. The marked and quick increase in INR values from 1.85 to 4.10 was observed on day 4 of the second cycle.

The Horn's Drug Interaction Probability Scale (19) indicated that the total score was 2, 4 and 4 in cases 1, 2 and 3, respectively. The scores indicate a possibility of drug interaction in each case.

Anticoagulant activity of warfarin. INR values prior to and following combination chemotherapy and the times to reach maximum values are shown in Table II. The mean value of 
Table I. Patient characteristics.

Patients

Characteristic

A

Age, years

Sex

Diagnosis

Type

Cardiac dysfunction

Prior therapy received (no. received)

Regimen

Dosage of lenalidomide, $\mathrm{mg}$

Treatment schedule, day

Dosage of dexamethasone, $\mathrm{mg}$

Treatment schedule, day

Enforced chemotherapy (cycle)

Analyzed chemotherapy (cycle)

Warfarin dose, mg

Additional administration of CYP2C9 inhibitors

Alteration of hepatic function (AST, ALT and T-Bil)

Alteration of serum albumin

Chemotherapy-induced nausea and appetite loss

70
Male

AL amyloidosis

BJP- $\lambda$

Non-sustained ventricular tachycardia Yes (1)

$$
\begin{gathered}
15 \\
1-21 \\
12
\end{gathered}
$$

$1,8,15,22,28$

1

1

4

No

No

No

No
B

71

Female

AL amyloidosis

BJP- $\lambda$

Complete

atrioventricular block

Yes (1)

15

$1-21$

12

$1,8,15,22,28$

2
1
1.5
No
No
No
No

71

Female

AL amyloidosis $\operatorname{IgG}-\lambda$

Sick sinus syndrome Yes (1)

40

$1,8,15,22,28$

3
2
2
No
No
No
No

AL, immunoglobulin light-chain; BJP, Bence Jones protein; CYP, cytochrome P450; AST, aspartate aminotransferase; ALT, glutamic-pyruvic transaminase; T-Bil, total bilirubin; IgG, immunoglobulin G.

A

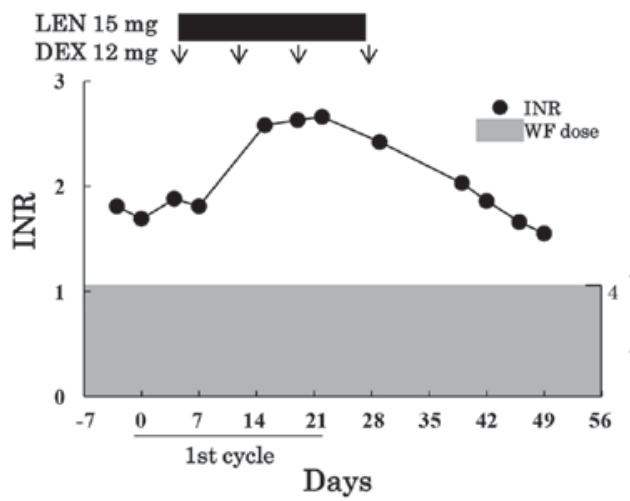

C

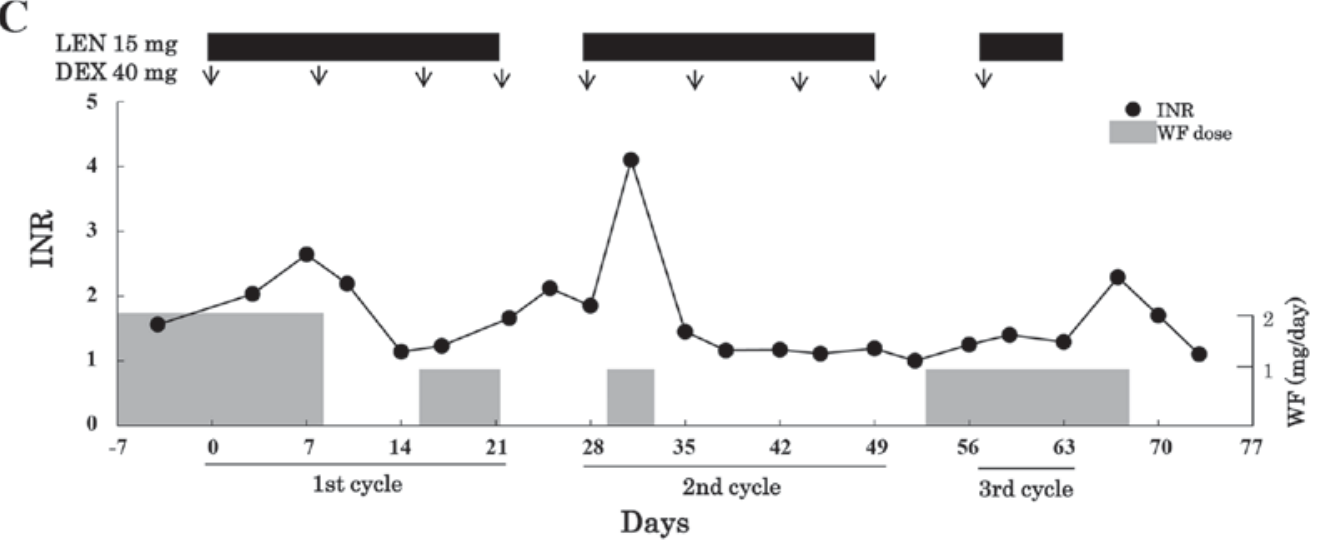

B

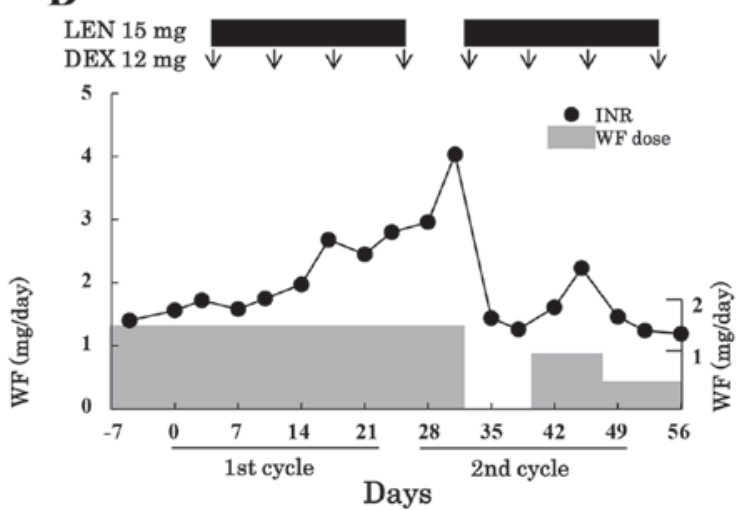

Days

Figure 1. Time courses of INR values and the doses of warfarin, lenalidomide and dexamethasone administered to patient (A) A, (B) B, and (C) C. LEN, lenalidomide; DEX, dexamethasone; WF, warfarin; INR, international normalized ratio. 
Table II. INR values prior to and following combination chemotherapy and times to reach its maximum values.

\begin{tabular}{lccc}
\hline & \multicolumn{2}{c}{$\begin{array}{c}\text { Combination } \\
\text { chemotherapy }\end{array}$} & \\
\cline { 2 - 3 } Parameter & Prior to & Subsequent to & P-value \\
\hline INR $^{\mathrm{a}}$ & $1.52 \pm 0.19$ & $2.60 \pm 0.22$ & 0.0003 \\
$\begin{array}{l}\text { Times to reach } \\
\text { maximum value, days }\end{array}$ & & $17(8-25)$ & \\
& & & \\
& & &
\end{tabular}

Among 6 cycles chemotherapy enforced in all patients, the data of 4 cycles were analysed. The other 2 cycles were excluded, because that the INR value prior to the combination chemotherapy was high as compared with the standard value (second cycle in Patient B), or warfarin was not administered prior to the combination chemotherapy (second cycle in Patient C). ${ }^{a}$ Mean \pm standard deviation, ${ }^{b}$ median (range). Comparison of data was performed with an unpaired Student's t-test. INR following concomitant use of lenalidomide, low-dose dexamethasone and warfarin was evaluated on maximum value. INR, international normalized ratio.

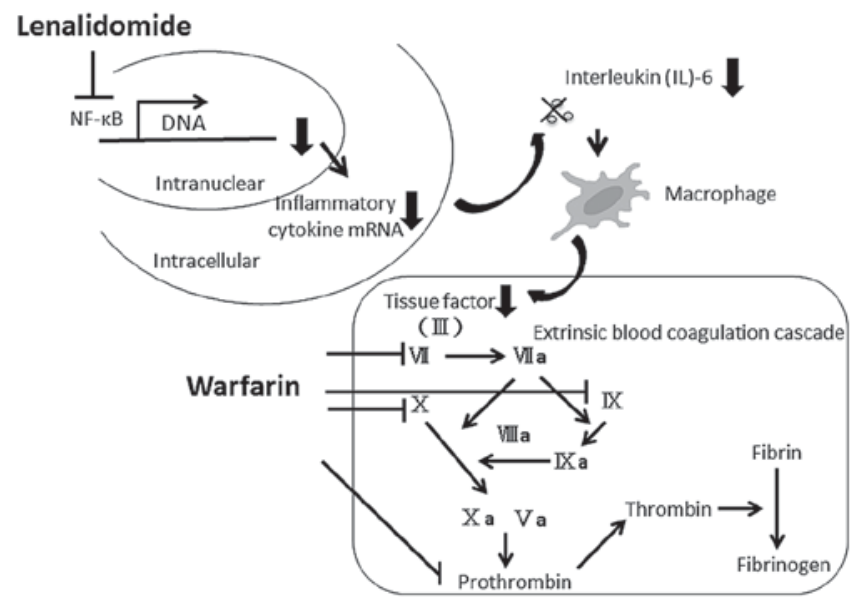

Figure 2. Postulated mechanism of the interaction between lenalidomide and warfarin. NF- $\kappa \beta$, nuclear Factor- $\kappa \beta$.

INR was 1.52 prior to the treatment and increased 1.7 -fold during combination chemotherapy $(\mathrm{P}=0.0003)$. The median time to reach the maximum value of INR was 17 days.

\section{Discussion}

In the present analysis, the additional use of warfarin with lenalidomide and low-dose dexamethasone combination therapy significantly increased the INR values. Therefore, this indicates a drug interaction between warfarin and the combination of lenalidomide and low-dose dexamethasone, which is of clinically importance.

The mechanism of the interaction between warfarin and combination therapy with lenalidomide and low-dose dexamethasone remains unclear, but a number of concepts were considered. With regards to the combination of warfarin and dexamethasone, it was reported that the high-dose of corticosteroids potentiated the effects of warfarin, resulting in increased INR values $(14,15)$. By contrast, Yano et al (17) reported that the increase in INR values was not observed with concomitant use of low-dose $(6.6 \mathrm{mg})$ dexamethasone, which is comparable to the results of the present analysis. The extent of interaction between warfarin and dexamethasone was poor, even though low-dose dexamethasone may increase INR values.

Lenalidomide is not metabolized by cytochrome P450 enzymes in the liver (8), and the ratio of binding to plasma protein was $\sim 40 \%$ (7). By contrast, warfarin is metabolized by CYP2C9 and has a high plasma protein binding ratio of 99\% (10-12). Therefore, there is unlikely to be a pharmacokinetic interaction between lenalidomide and warfarin. This was supported by the observation that concomitant use of multiple-dose lenalidomide with a single-dose warfarin had no effect on the pharmacokinetics of total lenalidomide or $\mathrm{R}$ - and S-warfarin in healthy volunteers (18). However, the alteration of INR values was time-dependent and reproducible for lenalidomide in the present analysis (Fig. 1). In addition, the increase in INR values continued, even though treatment with lenalidomide was discontinued (Fig. 1B), and increased markedly when lenalidomide was re-administered in the next cycle. The Horn's Drug Interaction Probability Scale (19) indicated that the interaction between warfarin and lenalidomide was a possibility. By contrast, patients exhibited no marked alterations in hepatic function and serum albumin concentration prior and subsequent to combination chemotherapy. Furthermore, and no additional administration of CYP2C9 inhibitors or vitamin $\mathrm{K}$ supplements was observed. In addition, chemotherapy-induced nausea or appetite loss, which may alter vitamin $\mathrm{K}$ absorption and serum albumin concentration, was not observed in any patients. These findings suggest that the total clearance or protein binding of warfarin remained unchanged. Accordingly, the combination of warfarin and lenalidomide was considered to result in pharmacodynamic interactions.

The mechanism of pharmacodynamic interaction between warfarin and lenalidomide was considered. Lenalidomide is known to have immunomodulatory effects, which alters the production of Th2-type cytokines, including IL-6 and tumor necrosis factor $\alpha$ and is primarily modulated by a decrease in IL-6 levels $(20,21)$. IL- 6 has been demonstrated to be associated with extrinsic blood coagulation cascades, which produces a tissue factor in macrophages (22). Lenalidomide may decrease the synthesis of a tissue factor by inhibiting the production of IL-6 and thus resulting in an increase of anticoagulant activity of warfarin, as shown in Fig. 2. The mechanism proposed by the authors of the present study was supported by a study into the drug interaction of capecitabine and warfarin, which demonstrated that capecitabine effects the factor VII activity and contributes to the increase in INR values (23). Collectively, the interaction between lenalidomide and warfarin may have occurred due to pharmacodynamic interaction by inhibiting the production of IL- 6 but not pharmacokinetic interaction. Analysis of levels of IL-6 and tissue factors may provide further insights into the precise mechanism of this interaction.

Clinically important interactions between warfarin and combination therapy with lenalidomide and low-dose dexamethasone were observed in AL amyloidosis, where there was a significant increase in INR values. Therefore, patients 
taking warfarin should be monitored closely and regularly for alterations in INR values during lenalidomide and low-dose dexamethasone combination therapy, and the dose of warfarin should be reduced if necessary.

\section{References}

1. Gertz MA: Immunoglobulin light chain amyloidosis: 2013 pdate on diagnosis, prognosis, and treatment. Am J Hematol 88: 416-425, 2013.

2. Lauta VM: A review of the cytokine network in multiple myeloma: Diagnostic, prognostic, and therapeutic implications. Cancer 97: 2440-2452, 2003.

3. Nagasawa T, Yanagisawa H, Hasegawa Y, Kanma H and Abe T: Polycythemia associated with primary systemic amyloidosis: Elevated levels of hemopoietic factors and cytokines. Am J Hematol 43: 57-60, 1993.

4. Sanchorawala V, Wright DG, Rosenzweig M, Finn KT, Fennessey S, Zeldis JB, Skinner M and Seldin DC: Lenalidomide and dexamethasone in the treatment of AL amyloidosis: Results of a phase 2 trial. Blood 109: 492-496, 2007.

5. Dimopoulos MA, Chen C, Spencer A, Niesvizky R, Attal M, Stadtmauer EA, Petrucci MT, Yu Z, Olesnyckyj M, Zeldis JB, et al: Long-term follow-up on overall survival from the MM-009 and MM-010 phase III trials of lenalidomide plus dexamethasone in patients with relapsed or refractory multiple myeloma. Leukemia 23: 2147-2152, 2009.

6. Palladini G and Comenzo RL: The challenge of systemic immunoglobulin light-chain amyloidosis (Al). Subcell Biochem 65 609-642, 2012

7. Chen N,Zhou S and Palmisano M: Clinical pharmacokinetics and pharmacodynamics of Lenalidomide. Clin Pharmacokinet 56: $139-152,2017$

8. Kumar G, Lau H and Laskin O: Lenalidomide: In vitro evaluation of the metabolism and assessment of cytochrome P450 inhibition and induction. Cancer Chemother Pharmacol 63: 1171-1175, 2009

9. Chen N, Weiss D, Reyes J, Liu L, Kasserra C, Wang X, Zhou S, Kumar G, Weiss L and Palmisano M: No clinically significant drug interactions between lenalidomide and P-glycoprotein substrates and inhibitors: Results from controlled phase I studies in healthy volunteers. Cancer Chemother Pharmacol 73: 1031-1039, 2014.

10. Holbrook AM, Pereira JA, Labiris R, McDonald H, Douketis JD, Crowther M and Wells PS: Systematic overview of warfarin and its drug and food interactions. Arch Intern Med 165: 1095-1106, 2005
11. Jones CB and Fugate SE: Levofloxacin and warfarin interaction. Ann Pharmacother 36: 1554-1557, 2002.

12. Greenblatt DJ and von Moltke LL: Interaction of warfarin with drugs, natural substances, and foods. J Clin Pharmacol 45: 127-132, 2005.

13. Strandell J and Wahlin S: Pharmacodynamic and pharmacokinetic drug interactions reported to VigiBase, the WHO global individual case safety report database. Eur J Clin Pharmacol 67: 633-641, 2011.

14. Sellam J, Costedoat-Chalumeau N, Amoura Z, Aymard G, Choquet S, Trad S, Vignes BL, Hulot JS, Berenbaum F, Lechat $\mathrm{P}$, et al: Potentiation of fluindione or warfarin by dexamethasone in multiple myeloma and AL amyloidosis. Joint Bone Spine 74: 446-452, 2007.

15. Kaufman M: Treatment of multiple sclerosis with high-dose corticosteroids may prolong the prothrombin time to dangerous levels in patients taking warfarin. Mult Scler 3: 248-249, 1997.

16. Chatterjea JB and Salomon L: Antagonistic effect of A.C.T.H. and cortisone on the anticoagulant activity of ethyl biscoumacetate. Br Med J 2: 790-792, 1954.

17. Yano R, Kurokawa T, Tsuyoshi H, Shinagawa A, Sawamura Y, Matsunaga A, Nakamura T, Yoshida Y, Yoneda M, Kotsuji F and Masada M: Transient elevation of international normalized ratio during cisplatin-based chemotherapy in patients who are taking warfarin. Ann Pharmacother 45: e55, 2011.

18. Weiss D, Knight R, Zhou S, Palmisano M and Chen N: Evaluation of pharmacokinetic and pharmacodynamic interactions when lenalidomide is co-administered with warfarin in a randomized clinical trial setting. Clin Drug Investig 35: 455-461, 2015.

19. Horn JR, Hansten PD and Chan LN: Proposal for a new tool to evaluate drug interaction cases. Ann Pharmacother 41: 674-680, 2007.

20. Quach H, Ritchie D, Stewart AK, Neeson P, Harrison S, Smyth MJ and Prince HM: Mechanism of action of immunomodulatory drugs (IMiDS) in multiple myeloma. Leukemia 24: 22-32, 2010.

21. Rushworth GF, Leslie SJ, Forsyth $\mathrm{P}$ and Vincent C: Evidence-based case report: Multiple thrombotic episodes associated with lenalidomide and dexamethasone therapy for multiple myeloma. Ther Adv Drug Saf 3: 115-122, 2012.

22. Ikeda U, Ito $T$ and Shimada K: Interleukin- 6 and acute coronary syndrome. Clin Cardiol 24: 701-704, 2001.

23. Camidge R, Reigner B, Cassidy J, Grange S, Abt M, Weidekamm E and Jodrell D: Significant effect of capecitabine on the pharmacokinetics and pharmacodynamics of warfarin in patients with cancer. J Clin Oncol 23: 4719-4725, 2005. 